\title{
Diseño hidráulico y evaluación a escala laboratorio de un sistema RBC (Rotating Biological Contactor) para tanque primario de aguas residuales no domésticas provenientes de una industria de bebidas no alcohólicas
}

\author{
Hydraulic design and laboratory-scale evaluation of a RBC \\ (Rotating Biological Contactor) system for primary non-domestic \\ wastewater tank from a non-alcoholic Beverage Industry
}

\author{
Luisa Fernanda Quintero Pulgar ${ }^{1} \quad$ Andrés Felipe Molano Guarín ${ }^{1} \quad$ Laura Mariela Pramparo ${ }^{1 *}$
}

Recibido 27 de marzo de 2019, aceptado 11 de marzo de 2020

Received: March 27, 2019 Accepted: March 11, 2020

\begin{abstract}
RESUMEN
Un sistema RBC (Rotating Biological Contactor) es una tecnología tipo BIO para tratamiento de aguas residuales, en la cual a partir de procesos de bioaumentación, los microorganismos presentes en el agua se fijan a unos discos rotativos parcialmente sumergidos y paralelos entre sí, formando una biopelícula [1]. Esta tecnología permite remover carga orgánica contaminante del agua, mejorando los procesos de tratamiento. En este documento, se presenta la evaluación a escala laboratorio de un sistema RBC, para aguas residuales de la industria de bebidas no alcohólicas, con el fin de reducir la tasa de generación de lodos residuales durante el tren de tratamiento, a través de oxidación y degradación de compuestos presentes en el agua. Para esto, se realizó la caracterización bioquímica del afluente, selección y bioaumentación de microorganismos aislados del agua residual y la determinación de parámetros hidráulicos de diseño óptimos para la evaluación del sistema en la escala laboratorio. Se encontró que el sistema es eficiente cuando opera con tiempos de retención hidráulica suficientes para una adecuada difusión de oxígeno y adherencia de la biopelícula, y que presenta porcentajes de remoción de $\mathrm{DQO}$ y $\mathrm{DBO}_{5}$ de más del $80 \%$ en cada una de sus etapas.
\end{abstract}

Palabras clave: Bioaumentación, biopelícula, carga orgánica contaminante, lodos, RBC.

\begin{abstract}
An RBC (Rotating Biological Contactor) system is a BIO type technology for wastewater treatment. From bioaugmentation processes, the present microorganism in the water is attached to partially submerged rotating discs and parallel to each other, forming a biofilm [1]. This technology allows removing contaminating organic load from the water, improving the treatment processes. In this document, the laboratory-scale evaluation of an RBC system is presented for wastewater from the non-alcoholic beverage industry to reduce the generation rate of residual sludge, during the treatment train, through oxidation and degradation of compounds present in water. For this purpose, the tributary's biochemical characterization, the selection and bioaugmentation of microorganisms isolated from the residual water, and the determination of optimal design of hydraulic parameters for the system evaluation in the laboratory-scale were carried out. It was found that the system is efficient when it operates with enough hydraulic retention times for adequate oxygen diffusion and adhesion of the biofilm and that it presents percentages of $D Q O$ and $D B O_{5}$ removal over $80 \%$ in each of its stages.
\end{abstract}

Keywords: Bioaugmentation, biofilm, contaminating organic load, sludge, RBC.

\footnotetext{
1 Universidad Militar Nueva Granada. Bogotá, Colombia.

E-mail: u1102164@unimilitar.edu.co; andres.molano@unimilitar.edu.co; laura.pramparo@unimilitar.edu.co

* Autor de correspondencia: laura.pramparo@unimilitar.edu.co
} 


\section{INTRODUCCIÓN}

En Colombia, la industria de bebidas no alcohólicas tiene una representación en el mercado del $56 \%$, está conformada por gaseosas, jugos de fruta, agua embotellada, té, bebidas hidratantes y energizantes. El consumo per cápita de estos productos depende de factores asociados a renta disponible y disminución de pobreza, y por esta razón su producción está en constante crecimiento [2]. Sumado a que la demanda de agua es uno de los factores más importantes por ser el insumo principal de elaboración (es uno de los mayores demandantes del recurso hídrico con aproximadamente $1300 \mathrm{~m}^{3} /$ día sólo en procesos de producción), aumenta la cantidad de aguas residuales que se generan, las cuales son ricas en compuestos nitrogenados, fosforados, azufrados, carbohidratos y sustancias biodegradables, producto de procesos como lavado de botellas y líneas de producción y de actividades de casinos y baterías de baños. El tratamiento de estas aguas residuales tiene impacto en los recursos hídricos, ya que la carga orgánica contaminante ronda los $1,7 \mathrm{KgDBO} / \mathrm{m}^{3}$, los cuales incluyen sólidos disueltos (orgánicos e inorgánicos) y sólidos suspendidos que son vertidos al sistema de alcantarillado o cuerpos de agua.

La mayoría de las plantas de tratamiento de aguas residuales industriales de sectores de alimentos y bebidas tienen como tecnología principal procesos biológicos (UASB, MBR, zanjones de oxidación, lodos activados, RBC, entre otros), donde previamente se realizan estabilizaciones químicas y manejo hidráulico como parte del tratamiento primario y se asegura de esta forma la eficiencia de los tratamientos biológicos [3]. En este caso, las aguas residuales llegan a una planta de tratamiento que cuenta con un tren conformado por operaciones de homogenización, ecualización, biofiltración, lodos activados y clarificación, donde los procesos aerobios y anaerobios son un componente integral para la degradación biológica. Sin embargo, las reacciones en los procesos de homogenización y ecualización generan lodos en el tratamiento primario que afectan las tasas de remoción y las operaciones del tratamiento secundario, así como costos adicionales de manejo al ser un residuo peligroso [4].

Las alternativas de tratamiento biológico suelen ser las más adecuadas para las aguas residuales con alto contenido de contaminantes orgánicos [5]. Su efectividad se ve influenciada por la mejora de la biodegradabilidad que se pueda implementar en el tratamiento primario que suele ser de tipo químico. La mayoría de estas alternativas están asociadas a procesos de descomposición de materia orgánica y separación de sólidos (biomasa).

Los biodiscos o contactores biológicos rotatorios son sistemas aerobios de biomasa adherida desarrollados a inicios del siglo XX para el tratamiento biológico del agua residual doméstica; sin embargo, su comercialización, investigación e instalación se presentó en 1970 en Alemania y Estados Unidos [6] y se ha convertido en una buena opción para el tratamiento de aguas residuales domésticas de pequeñas comunidades [7]. En Colombia se han realizado diversos estudios donde se ha planteado este tipo de técnica como alternativa de tratamiento biológico y se han obtenido buenos resultados para la remoción de carga orgánica $[8,9,10]$.

Por lo anterior, y teniendo en cuenta la problemática asociada a generación de lodos en la fase primaria de la planta de tratamiento de la industria de bebidas no alcohólicas, se evalúa en el laboratorio el sistema RBC (Rotating Biological Contactor) con aguas residuales provenientes de dicha industria, para una futura reingeniería del sistema de tratamiento actual mediante este tipo de biotecnología, el cual consiste en la remoción de materia orgánica del agua a través de discos unidos por un eje y paralelos entre sí, sumergidos parcialmente en el agua residual. En el sistema se dan procesos de bioaumentación que crean el ambiente adecuado para permitir la adherencia de microorganismos presentes y la formación de biopelícula alrededor de los discos [1]. Esto con el fin de aumentar las tasas de remoción de lodos y mejorar la eficiencia de los procesos de la planta mediante un biotratamiento de aguas residuales no domésticas.

En este artículo se presenta el diseño hidráulico de un modelo a escala laboratorio de la tecnología RBC y su evaluación para el tratamiento de aguas residuales de una industria de bebidas no alcohólicas.

\section{METODOLOGÍA}

Para llevar a cabo la evaluación del sistema RBC, se realizó una primera fase que comprendió la 
caracterización del afluente para obtener datos de entrada en el sistema. Esta caracterización se realizó con muestras obtenidas de una planta de tratamiento de la industria de bebidas no alcohólicas, en las operaciones de ecualización, homogenización y biofiltro, siendo este último la opción contemplada para la reingeniería a futuro con el sistema RBC. Los parámetros de calidad empleados para caracterizar en esta fase fueron: Oxígeno Disuelto (OD) por el método Winkler, Demanda Bioquímica de Oxígeno a 5 días $\left(\mathrm{DBO}_{5}\right)$ con método de incubación y electrometría y Demanda Química de Oxígeno (DQO) con método de reflujo cerrado. Todos los parámetros se realizaron bajo los protocolos del Instituto de Hidrología, Meteorología y Estudios Ambientales de Colombia (IDEAM), TP0084, TP0087 y TP0086, respectivamente [11].

La siguiente fase consistió en evaluar los porcentajes de remoción de materia orgánica de dos consorcios microbianos aislados de las aguas residuales de la industria de bebidas no alcohólicas, con base en los parámetros de calidad evaluados en la etapa de caracterización $\left(\mathrm{DBO}_{5}\right.$ y DQO). En este caso, para cada consorcio se evaluaron muestras de 24 , 48 y 72 horas, con y sin centrifugación, con el fin de determinar eficiencia al aumentar la biomasa.

Posteriormente, se realizó la inmovilización de los microorganismos seleccionados en la fase de evaluación de consorcios, en los discos del sistema a escala laboratorio. El sustrato inmovilizador utilizado fue Alginato de Sodio de algas café y la fijación fue hecha con Cloruro de Calcio anhidro.

El sistema RBC evaluado en el laboratorio cuenta con 4 etapas, cada una con siete discos separados 1 centímetro entre sí, los discos tienen un diámetro de 23 centímetros y un espesor de 0,5 centímetros. De esta forma, se tiene un área efectiva en el sistema de $2,31 \mathrm{~m}^{2}$. Cada etapa tiene longitud de 23,5 centímetros, para una longitud total del reactor de 0,94 metros. El porcentaje de área sumergida de los discos es del $40 \%[12,13,14]$ y el volumen efectivo del reactor es de 21 litros aproximadamente. La velocidad de rotación de los discos se estableció en $40 \mathrm{rpm}$, ya que ésta garantiza buena oxigenación de los discos. El reactor está hecho de acero inoxidable para evitar oxidación de las partes por la composición del agua residual tratada, de igual forma, los discos son de corte de metal y textura rugosa, lo que permite una mejor adherencia de la película.

Se realizaron cuatro corridas del sistema con diferentes tiempos de retención hidráulica y con diferentes cepas inmovilizadas. Se tomaron muestras de cada etapa al finalizar la operación y se evaluaron los parámetros de calidad ya mencionados (OD, $\mathrm{DBO}_{5} \mathrm{Y}$ DQO).

\section{RESULTADOS Y ANÁLISIS}

\section{Caracterización bioquímica de las aguas residuales de Industria de Bebidas no alcohólicas (DQO vs DBO)}

En la Tabla 1 se presentan los resultados obtenidos de las determinaciones de carga contaminante, tanto orgánica como inorgánica, por medio de la medición de $\mathrm{DBO}_{5}$ y $\mathrm{DQO}$ en las Operaciones Unitarias (Ecualizador, Homogenizador, Biofiltro) y Blanco, siendo este último usado como medida de control.

Según los resultados que se presentan en la Tabla 1, se determina que la actividad con mayor concentración de carga contaminante fue el Biofiltro. Estos datos se deben a la concentración acumulada de biopelículas que se tienen en el sistema de biofiltro que no han sido removidas por parte de la empresa y que el material de fijación permitió el crecimiento de comunidades microbianas aumentando los procesos metabólicos que aportan productos de tipo orgánico e inorgánico.

Teniendo en cuenta lo observado en la Figura 1, la medida de la DQO en la operación unitaria Ecualizador es de $2304 \mathrm{mg} / \mathrm{L}$ como fuente de entrada y al pasar por la operación unitaria Homogenizador es $780,8 \mathrm{mg} / \mathrm{L}$, se tiene una remoción de Carga contaminante del $66 \%$. Sin embargo, en la operación unitaria Biofiltro aumentó un $31 \%$ más de carga

Tabla 1. Caracterización Bioquímica.

\begin{tabular}{|l|c|c|}
\hline \multicolumn{1}{|c|}{ Muestra } & $\begin{array}{c}\text { DQO } \mathbf{~ m g O}_{\mathbf{2}} / \mathbf{L} \\
\text { (Determinada) }\end{array}$ & $\begin{array}{c}\mathbf{D B O}_{\mathbf{5}} \mathbf{~ m g O}_{\mathbf{2}} / \mathbf{L} \\
\text { (Determinada) }\end{array}$ \\
\hline Ecualizador & 2304 & 492,7 \\
\hline Homogenizador & 780,8 & 174,2 \\
\hline Biofiltro & 1024 & 881,2 \\
\hline Blanco & 0 & 8,61 \\
\hline
\end{tabular}

Fuente: Los autores, 2018. 
Fuente: Los autores, 2018.

Caracterización Bioquímica del Afluente

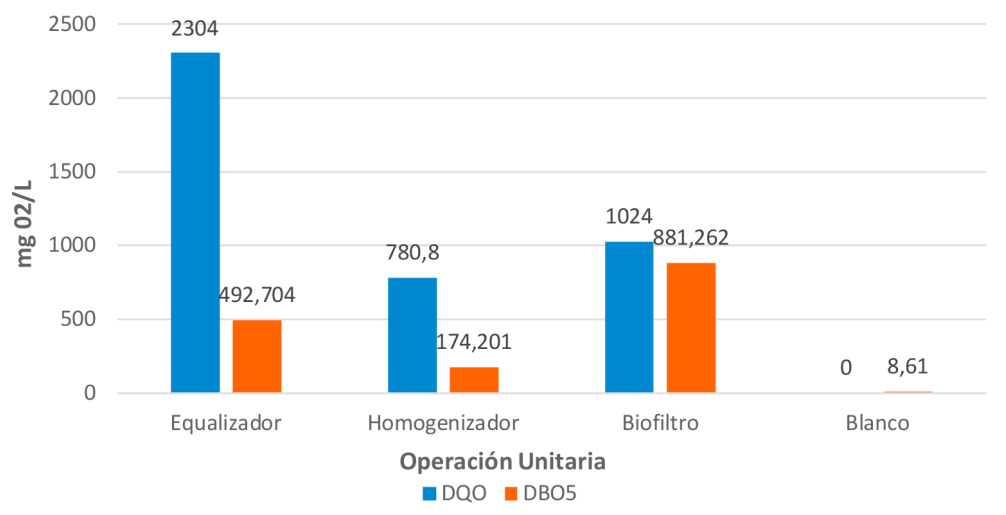

Figura 1. Caracterización Bioquímica del afluente.

orgánica contaminante, lo que genera un balance negativo al ingreso de la operación unitaria Lodos Activados y a su vez da como resultado el aumento de los lodos creando así condiciones de anaerobiosis. Por esta razón, se selecciona como prueba las aguas residuales provenientes de la operación Biofiltro usando como reingeniería en el proceso el sistema RBC (Rotating Biological Contactor).

A un biofiltro llegan aguas residuales que han sido sometidas a un tratamiento primario previo, allí se eliminan sólidos que puedan quedar de las operaciones de pretratamiento y se proporciona la superficie donde crecen los microorganismos encargados de degradar aeróbica y anaeróbicamente la carga orgánica contaminante. Los sólidos orgánicos suspendidos son retenidos en el lecho filtrante y los constituyentes orgánicos son mineralizados por las bacterias. De igual forma, también existe remoción de nitrógeno por parte de los microorganismos, a través de nitrificación-desnitrificación [15].

\section{Selección y evaluación de cepas y consorcios microbianos}

En la Fase I del proyecto de investigación enfocado en la bioprospección de microorganismos en aguas residuales, se obtuvieron aislamientos de diferentes morfotipos de microorganismos y la determinación de pruebas enzimáticas (Hidrolíticas, Proteolíticas y Lipolíticas). Estos microorganismos se seleccionaron y fueron puestos en $150 \mathrm{~mL}$ de aguas residuales provenientes de la operación unitaria Biofiltro, de esta forma se seleccionaron las cepas con mayor remoción de DQO y DBO en forma aislada. Estas se denominaron de la siguiente manera: "CEPA271", "CEPA-277”, “CEPA-342”, "CEPA-346”, "CEPA-261" y "CEPA-342", las cuales fueron identificadas molecularmente.

De esta forma se inició el proceso de evaluación de comportamiento mediante la técnica de enfrentamiento en la caja de Petri sobre sustrato nutritivo, la cual consiste en una siembra en medio sólido colocando un microorganismo frente a otro, para verificar que al crecer no produzcan sustancias que inhiban el crecimiento. Con base en esto, se seleccionaron los que no presentan dicha inhibición ya que en el sistema planteado se necesita que se forme una biopelícula, y los microorganismos no deben competir por espacio físico ni generar dichas sustancias, para que puedan vivir en una misma área.

Las pruebas dieron como resultado las siguientes combinaciones para iniciar las pruebas de remoción de carga contaminante $\left(\mathrm{DQO}\right.$ y $\mathrm{DBO}_{5}$ ) teniendo como referencia el valor obtenido de $\mathrm{DQO}$ y $\mathrm{DBO}_{5}$ en la operación unitaria Biofiltro (ver Tabla 2). Estas pruebas se realizaron en volúmenes de $250 \mathrm{~mL}$ usando como sustrato aguas residuales provenientes de la operación unitaria Biofiltro, con tiempos de retención celular de 24, 48 y 72 horas a una concentración de $1 \times 10^{8} \mathrm{UFC} / \mathrm{mL}$. Los mejores resultados se muestran en la siguiente Tabla.

Es bien conocido que la Demanda Química de Oxígeno (DQO) corresponde a la cantidad de oxígeno 
Tabla 2. Consorcios Microbianos.

\begin{tabular}{|c|c|c|c|c|c|}
\hline \multirow{6}{*}{$\begin{array}{l}\text { CONSORCIO } 1 \\
\text { BNA } 261, \\
\text { BNA241, } \\
\text { BNA } 277, \\
\text { BNA } 342, \\
\text { BNA } 346\end{array}$} & $\begin{array}{l}\text { Muestra Sin } \\
\text { Centrifugar }\end{array}$ & $\begin{array}{c}\mathrm{DQO}\left(\mathrm{mgO}_{2} / \mathrm{L}\right) \\
\text { Determinada }\end{array}$ & $\begin{array}{l}\text { Porcentaje de } \\
\text { remoción }\end{array}$ & $\begin{array}{c}\mathrm{DBO}_{5}\left(\mathrm{mgO}_{2} / \mathrm{L}\right) \\
\text { Determinada }\end{array}$ & $\begin{array}{l}\text { Porcentaje } \\
\text { de remoción }\end{array}$ \\
\hline & $72 \mathrm{R} 1$ & 300,8 & 70,62 & 268,1 & 69,6 \\
\hline & Blanco & 0 & 0 & 0 & 0 \\
\hline & $\begin{array}{c}\text { Muestra } \\
\text { Centrifugado }\end{array}$ & $\begin{array}{c}\mathrm{DQO}\left(\mathrm{mgO}_{2} / \mathrm{L}\right) \\
\text { Determinada }\end{array}$ & $\begin{array}{l}\text { Porcentaje de } \\
\text { remoción }\end{array}$ & $\begin{array}{c}\mathrm{DBO}_{5}\left(\mathrm{mgO}_{2} / \mathrm{L}\right) \\
\text { Determinada }\end{array}$ & $\begin{array}{l}\text { Porcentaje } \\
\text { de remoción }\end{array}$ \\
\hline & $48 \mathrm{R} 1$ & 664,4 & 35,12 & 326,5 & 63,0 \\
\hline & Blanco & 0 & 0 & 0 & 0 \\
\hline \multirow{6}{*}{$\begin{array}{c}\text { CONSORCIO } 2 \\
\text { BNA 49, 59, } \\
19,105,154, \\
255,106,278\end{array}$} & $\begin{array}{l}\text { Muestra Sin } \\
\text { Centrifugar }\end{array}$ & $\begin{array}{c}\mathrm{DQO}\left(\mathrm{mgO}_{2} / \mathrm{L}\right) \\
\text { Determinada }\end{array}$ & $\begin{array}{l}\text { Porcentaje de } \\
\text { remoción }\end{array}$ & $\begin{array}{c}\mathrm{DBO}_{5}\left(\mathrm{mgO}_{2} / \mathrm{L}\right) \\
\text { Determinada }\end{array}$ & $\begin{array}{l}\text { Porcentaje } \\
\text { de remoción }\end{array}$ \\
\hline & $48 \mathrm{R} 1$ & 675,3 & 34,1 & 356,3 & 59,6 \\
\hline & Blanco & 0 & 0 & 0 & 0 \\
\hline & $\begin{array}{c}\text { Muestra } \\
\text { Centrifugado } \\
\end{array}$ & $\begin{array}{c}\mathrm{DQO}\left(\mathrm{mgO}_{2} / \mathrm{L}\right) \\
\text { Determinada }\end{array}$ & $\begin{array}{l}\text { Porcentaje de } \\
\text { remoción }\end{array}$ & $\begin{array}{c}\mathrm{DBO}_{5}\left(\mathrm{mgO}_{2} / \mathrm{L}\right) \\
\text { Determinada } \\
\end{array}$ & $\begin{array}{l}\text { Porcentaje } \\
\text { de remoción }\end{array}$ \\
\hline & $24 \mathrm{R} 2$ & 656,4 & 35,9 & 339,9 & 61,5 \\
\hline & Blanco & 0 & & 0 & 0 \\
\hline
\end{tabular}

Fuente: Los autores, 2018.

requerida para oxidar químicamente los compuestos orgánicos presentes en el agua residual a $\mathrm{CO}_{2} \mathrm{y} \mathrm{H}_{2} \mathrm{O}$; la Demanda Bioquímica de Oxígeno $\left(\mathrm{DBO}_{5}\right)$, por su parte, es la cantidad de oxígeno usado por los microorganismos para metabolizar los compuestos orgánicos degradables como Carbohidratos, Proteínas, Hidrocarburos, Grasas y aceites y convertirlos en $\mathrm{CO}_{2}, \mathrm{H}_{2} \mathrm{O}, \mathrm{NH}_{4}$, minerales y biomasa [16].

En el caso de la industria de bebidas no alcohólicas, la concentración inicial de $\mathrm{O}_{2}$ en las aguas residuales de la operación unitaria Biofiltro fue $0,453 \mathrm{mg} / \mathrm{L}$, al colocarse en volúmenes de $250 \mathrm{~mL}$, con tiempos de retención celular 24, 48 y 72 horas y a una velocidad de agitación 120 rpm, se tenía mayor difusión de oxígeno. Esto indica que los microorganismos son de carácter facultativo, donde los procesos metabólicos se adaptan a la concentración de oxígeno disuelto para obtener una mayor remoción de carga orgánica contaminante. Por esta razón, se seleccionó el consorcio 1 centrifugado con tiempo de retención celular de 48 horas, el cual generó una concentración celular de $1 * 10^{6} \mathrm{UFC} / \mathrm{mL}$ de manera constante, lo que representó unas condiciones ideales de crecimiento microbiano diferentes a las que se presentan en 24 horas, condición en la que se podría estar generando un aumento en la concentración de $\mathrm{CO}_{2}$ por los procesos de metabolismo microbiano, que generan una disminución en la concentración celular (ver porcentaje de remoción de $\mathrm{DBO}_{5}$ ).

La selección de consorcios se basó en aquellos que fueron centrifugados, ya que presentaron mejores tasas de remoción que las muestras sin centrifugar. Con este procedimiento se remueve en cierta forma carga orgánica contaminante al separar la biomasa microbiana, ya que se obtienen dos fases visibles en las muestras: la primera de biomasa y sólidos y la segunda el sobrenadante, en el cual quedan los compuestos después de la biodegradación, tales como amonio, nitratos, nitritos, biomasa residual, entre otros. Este procedimiento de centrifugación se llevó a cabo asociando la operación del sistema $\mathrm{RBC}$, cuyos discos ejercen la fuerza giratoria sobre un mismo eje separando sólidos y biomasa del agua residual, los cuales por acción de la gravedad quedan en el fondo del sistema [1]. Teniendo en cuenta que los microorganismos se fijaron a los discos del sistema RBC, al centrifugar se buscó simular una fijación de la biomasa.

Las tasas de retención celular más adecuadas se seleccionan de acuerdo al crecimiento de los microorganismos, a los cuales se les debe asegurar un tiempo de permanencia suficiente para que se reproduzcan [17]. Este crecimiento se ve afectado 
por la velocidad a la que los microorganismos metabolizan el sustrato, y tiene unas fases que son: Estacionaria, en la cual los microrganismos se adaptan al medio, y comienzan a dividirse, Logarítmica-Exponencial, en la cual la velocidad de crecimiento es alta ya que los microorganismos se duplican en poco tiempo, Estacionaria, donde la población no aumenta debido a agotamiento de sustrato o igualación de células nuevas con células muertas y Lisis, donde son más células muertas que las producidas y la población disminuye [18]. En aguas residuales, estas fases influyen en la concentración celular de los consorcios microbianos que se seleccionan para llevar a cabo la degradación de materia orgánica, el tiempo de retención celular indica la fase en la que se encuentra la población microbiana y su efectividad en el metabolismo de compuestos orgánicos presentes en las aguas residuales mediante la actividad bioquímica de los microorganismos [19].

Por esta razón, las pruebas de inoculación de los dos consorcios realizadas en Erlenmeyer de $250 \mathrm{~mL}$, con diferentes tiempos de retención celular $(24,48$ y 72 horas), permitieron identificar las diferentes concentraciones celulares de acuerdo a las etapas de crecimiento bacteriano ya mencionadas $\left(1 \times 10^{4}\right.$, $1 \times 10^{6}$, y 1 x $10^{8} \mathrm{UFC} / \mathrm{mL}$ respectivamente) y sus diferentes tasas de remoción, seleccionando la de mayor actividad bioquímica (o mayor remoción) y así llevarlas a inmovilizar.

En este caso, al fijar el consorcio seleccionado en los biodiscos con un polímero, se mantenía la concentración celular de $1 \times 10^{6} \mathrm{UFC} / \mathrm{mL}$, y los microorganismos al estar inmovilizados tenían un tiempo de retención celular equivalente al tiempo de retención hidráulica del sistema.

\section{Puesta en marcha del sistema RBC}

Se procedió con las marchas en el sistema RBC mostrado en la Figura 2, el cual contaba con las siguientes especificaciones de diseño: a. $0,940 \mathrm{~m}$ de largo, 0,34 $\mathrm{m}$ de ancho, 0,17 $\mathrm{m}$ de Profundidad, 4 etapas cada una con 7 discos de $0,23 \mathrm{~m}$ de diámetro, separados a $0,01 \mathrm{~m}$ de distancia entre sí y a $0,07 \mathrm{~m}$ entre etapas, Eje transversal de $1,10 \mathrm{~m}$ en acero inoxidable, b. Caudales de diseño de $0,00019 \mathrm{~m}^{3} / \mathrm{h}$, c. Tiempo de Retención Hidráulica de 112 horas y cada etapa con un volumen de 5,325 Litros, para un volumen total de tratamiento de
21,3 Litros, d. Velocidad de rotación de discos de $40 \mathrm{rpm}$.

De acuerdo a estas dimensiones y según [20,21, 22 y 23], se procedió a realizar cuatro corridas, cada una con diferentes tiempos de operación, pero mismo volumen de tratamiento. En la Tabla 3 se presentan los resultados más significativos en cuanto a las determinaciones de $\mathrm{DBO}_{5}$ y DQO mediante dos réplicas por cada etapa.

En la Tabla anterior se observa que la Etapa 2 del tratamiento durante una operación de dos horas tuvo una remoción mínima de DQO del 12,5\% y una remoción del 84,6\% en $\mathrm{DBO}_{5}$, esto puede deberse a que los tiempos de retención celular no fueron los óptimos y el tiempo de retención hidráulica fue muy poco para tener contacto entre la biopelícula que contenía todas las cepas evaluadas como un solo consorcio, es importante evaluar que los compuestos inorgánicos de este tipo de efluente son variados y se necesita un mayor tiempo de retención hidráulica para que los discos aumenten la concentración de oxígeno y así poder oxidar este tipo de compuestos.

Fuente: Los autores, 2018.

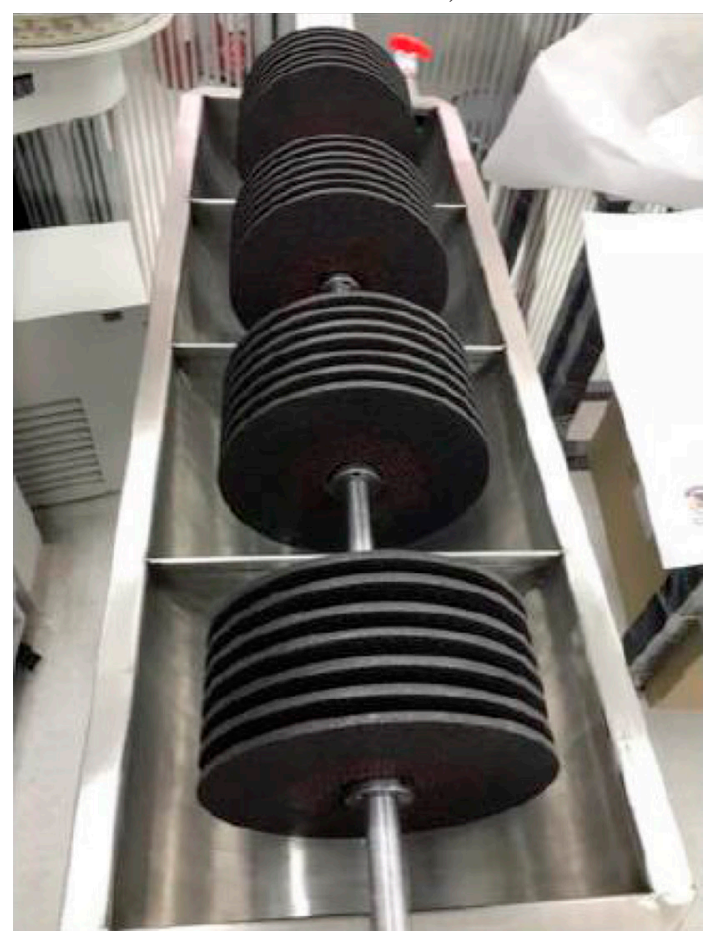

Figura 2. Prototipo a escala laboratorio. 
Tabla 3. Corrida 1 con Cepas 271-277-342-346-261-342.

\begin{tabular}{|c|c|c|c|c|c|c|}
\hline \multirow{6}{*}{$\begin{array}{c}\text { CORRIDA } 1 \text { CEPAS: } \\
271,277,342,346, \\
261,342\end{array}$} & \multicolumn{2}{|c|}{ Muestra } & $\begin{array}{c}\mathrm{DQO}\left(\mathrm{mg} \mathrm{O}_{2} / \mathrm{L}\right) \\
\text { Determinada }\end{array}$ & $\begin{array}{c}\% \\
\text { remoción }\end{array}$ & $\begin{array}{c}\mathrm{DBO}_{5}\left(\mathrm{mg} \mathrm{O} \mathrm{O}_{2} / \mathrm{L}\right) \\
\text { Determinada }\end{array}$ & $\begin{array}{c}\% \\
\text { remoción }\end{array}$ \\
\hline & \multirow{2}{*}{ Blanco } & R1 & 0 & 0 & 0 & 0 \\
\hline & & $\mathrm{R} 2$ & 0 & 0 & 0 & 0 \\
\hline & \multirow{2}{*}{ Afluente } & $\mathrm{R} 1$ & 1024 & 0 & 881,2 & 0 \\
\hline & & $\mathrm{R} 2$ & 994 & 0 & 870 & 0 \\
\hline & Etapa 2 & R1 & 896 & 12,5 & 135,9 & 84,6 \\
\hline
\end{tabular}

Fuente: Los autores, 2018.

En la Tabla 4, se observa una remoción de DQO en las tres etapas del sistema de más del $60 \%$ respecto a los que se muestran en la Tabla 3. Esto confirma que, al aumentar el tiempo de operación del sistema a 3 horas, se obtiene una mejor difusión de oxígeno permitiéndole a los microorganismos oxidar los compuestos del afluente y tener una mejor fijación al biodisco. De igual forma, la separación del consorcio utilizando sólo 3 cepas pudo influir en un mejor comportamiento de las comunidades permitiendo la fijación al biodisco ya nombrada.
En la Tabla 5, se presentan los datos correspondientes a la tercera corrida del sistema, la cual tuvo un tiempo de operación de 3 horas y un rango de porcentajes de remoción de $\mathrm{DQO}$ en el que se encuentran también los datos de DQO de la corrida 2 (ver Tabla 4), de esta forma se confirma la influencia del tiempo de retención hidráulica en la eficiencia del sistema y en el tiempo de retención celular, el cual se ve reflejado en los valores de remoción de $\mathrm{DBO}_{5}$. Sin embargo, la DQO y DBO no son constantes en las tres etapas, ya que en la etapa 2 no se obtuvo un desempeño adecuado.

Tabla 4. Corrida 2 con Cepas 271-277-342.

\begin{tabular}{|c|c|c|c|c|c|c|}
\hline \multirow{8}{*}{$\begin{array}{c}\text { CORRIDA } 2 \text { CEPAS: } \\
271,277,342\end{array}$} & \multicolumn{2}{|c|}{ Muestra } & $\begin{array}{c}\mathrm{DQO}\left(\mathrm{mgO}_{2} / \mathrm{L}\right) \\
\text { Determinada }\end{array}$ & $\begin{array}{c}\% \\
\text { remoción }\end{array}$ & $\begin{array}{c}\mathrm{DBO}_{5}\left(\mathrm{mgO}_{2} / \mathrm{L}\right) \\
\text { Determinada }\end{array}$ & $\begin{array}{c}\% \\
\text { remoción }\end{array}$ \\
\hline & \multirow{2}{*}{ Blanco } & R1 & 0 & 0 & 0 & 0 \\
\hline & & R2 & 0 & 0 & 0 & 0 \\
\hline & \multirow{2}{*}{ Afluente } & $\mathrm{R} 1$ & 1024 & 0 & 884,1 & 0 \\
\hline & & $\mathrm{R} 2$ & 944 & 0 & 807,4 & 0 \\
\hline & Etapa 1 & $\mathrm{R} 2$ & 243,2 & 76,3 & 101,1 & 88,6 \\
\hline & Etapa 2 & $\mathrm{R} 2$ & 281,6 & 72,5 & 146,1 & 83,5 \\
\hline & Etapa 3 & R1 & 256 & 75,0 & 106,5 & 88,0 \\
\hline
\end{tabular}

Fuente: Los autores, 2018.

Tabla 5. Corrida 3 con Cepas 346-261-342.

\begin{tabular}{|c|c|c|c|c|c|c|}
\hline \multirow{2}{*}{} & \multicolumn{2}{|c|}{ Muestra } & $\begin{array}{c}\text { DQO }\left(\mathbf{m g O}_{2} / \mathbf{L}\right) \\
\text { Determinada }\end{array}$ & $\begin{array}{c}\text { \% } \\
\text { remoción }\end{array}$ & $\begin{array}{c}\mathbf{D B O}_{\mathbf{5}}\left(\mathbf{m g O}_{2} / \mathbf{L}\right) \\
\text { Determinada }\end{array}$ & $\begin{array}{c}\% \\
\text { remoción }\end{array}$ \\
\cline { 2 - 7 } CORRIDA 3 CEPAS: & \multirow{2}{*}{$\begin{array}{c}\text { Blanco } \\
\text { 346, 261, 342 }\end{array}$} & $\mathrm{R} 1$ & 0 & 0 & 0 & 0 \\
\cline { 2 - 7 } & $\mathrm{R} 2$ & 0 & 0 & 0 & 0 \\
\cline { 2 - 7 } & Afluente & $\mathrm{R} 1$ & 1024 & 0 & 884,1 & 0 \\
\cline { 2 - 7 } & $\mathrm{R} 2$ & 944 & 0 & 807,4 & 0 \\
\cline { 2 - 7 } & Etapa 1 & $\mathrm{R} 1$ & 428 & 58,3 & 78,3 & 91,2 \\
\cline { 2 - 7 } & Etapa 3 & $\mathrm{R} 1$ & 384 & 62,5 & 89,7 & 89,9 \\
\hline
\end{tabular}

Fuente: Los autores, 2018. 
Para un tiempo de retención hidráulica de 7 horas, se obtienen los valores mostrados en la Tabla 6 . Se observa una mayor remoción de DQO a medida que el agua residual avanza por las etapas del sistema como se muestra en la Figura 3. Con los valores obtenidos tanto de $\mathrm{DBO}_{5}$ como de DQO, se confirma que, al aumentar el tiempo de operación del sistema, se obtiene una mejor oxidación de los compuestos del afluente, lo que permite también la degradación de materia orgánica, la cual es abundante en este tipo de aguas residuales. Esto indica que el sistema remueve carga orgánica con éxito al diseñar con tiempos de retención hidráulica mayores, en este caso las siete horas permitieron una mejor difusión de oxígeno y a su vez una disminución de valores de los parámetros de calidad evaluados.

\section{CONCLUSIONES}

Se realizó la caracterización bioquímica del agua residual proveniente de la planta de tratamiento de una industria de bebidas no alcohólicas. De esta forma, se determinaron los valores correspondientes de DQO y DBO para las operaciones unitarias ecualizador, homogenizador y biofiltro. Se encontró que en esta última operación existe un aumento en la concentración de carga contaminante, mediante valores de DQO y $\mathrm{DBO}_{5}$ de 1024 y $881,2 \mathrm{mg} \mathrm{O}_{2} / \mathrm{L}$ respectivamente, lo cual genera problemas en el tren de tratamiento asociados a la producción de lodos.

Con base en la caracterización realizada, se seleccionaron las aguas residuales de la operación

Tabla 6. Corrida 4 con Cepas 342-271-261.

\begin{tabular}{|c|c|c|c|c|c|c|}
\hline \multirow{8}{*}{$\begin{array}{c}\text { CORRIDA } 4 \text { CEPAS: } \\
342,271,261\end{array}$} & \multicolumn{2}{|c|}{ Muestra } & $\begin{array}{c}\mathrm{DQO}\left(\mathrm{mgO}_{2} / \mathrm{L}\right) \\
\text { Determinada }\end{array}$ & $\begin{array}{c}\% \\
\text { remoción }\end{array}$ & $\begin{array}{c}\mathrm{DBO}_{5}\left(\mathrm{mgO}_{2} / \mathrm{L}\right) \\
\text { Determinada }\end{array}$ & $\begin{array}{c}\% \\
\text { remoción }\end{array}$ \\
\hline & \multirow{2}{*}{ Blanco } & R1 & 0 & 0 & 0 & 0 \\
\hline & & R2 & 0 & 0 & 0 & 0 \\
\hline & \multirow{2}{*}{ Afluente } & R1 & 1024 & 0 & 884,1 & 0 \\
\hline & & $\mathrm{R} 2$ & 944 & 0 & 807,4 & 0 \\
\hline & Etapa 1 & R2 & 76,6 & 91,9 & 33,4 & 95,9 \\
\hline & Etapa 2 & R1 & 63,5 & 93,3 & 32,5 & 96,0 \\
\hline & Etapa 3 & $\mathrm{R} 2$ & 60,0 & 93,6 & 30,2 & 96,3 \\
\hline
\end{tabular}

Fuente: Los autores, 2018.

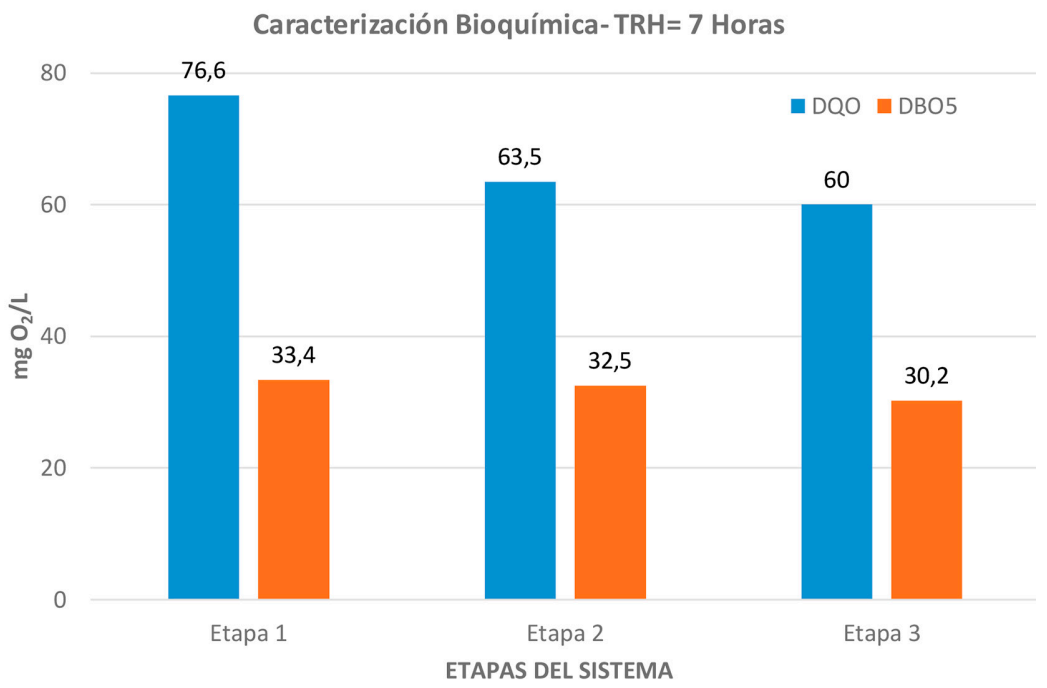

Figura 3. Resultados de operación para tiempo de retención hidráulica 7 horas. 
unitaria biofiltro, como muestra para la evaluación a escala laboratorio del sistema RBC. Se encontró un mejor comportamiento asociado a una mayor remoción de carga orgánica con el Consorcio 1 Centrifugado (BNA 261, BNA 241, BNA 277, BNA 342 y BNA 346), a un tiempo de retención celular de 48 horas y una concentración celular de $1 * 10^{6} \mathrm{UFC} / \mathrm{mL}$.

Se realizaron las pruebas a escala laboratorio del sistema RBC, obteniendo porcentajes de remoción de $\mathrm{DQO}$ y $\mathrm{DBO}_{5}$ de hasta 93 y $96 \%$ respectivamente, con tiempos de retención hidráulica y celular de 7 horas y velocidad de rotación de discos $40 \mathrm{rpm}$. De esta forma, se comprueba la eficiencia del sistema en la degradación de compuestos orgánicos de las aguas residuales provenientes de una industria de bebidas no alcohólicas. Con esta remoción de carga orgánica, se reduce la tasa de generación de lodos residuales mediante la bioaumentación e inmovilización de microorganismos aislados de las aguas residuales de la industria, en el sistema $\mathrm{RBC}$, bajo los parámetros hidráulicos de diseño trabajados.

\section{AGRADECIMIENTOS}

Producto derivado del proyecto IMP-ING-2655 financiado por la Vicerrectoría de Investigaciones de la Universidad Militar Nueva Granada, vigencia 2019.

\section{REFERENCIAS}

[1] L. Wang, N. Pereira and Y.-T. Hung. "Biological Treatment Processes". New York, USA. Humana Press. 2009.

[2] LEGISCOMEX. "Informe Sectorial- Bebidas no alcohólicas en Colombia”. Legiscomex, Bogotá. 2014.

[3] M. Matosic, I. Prstec, H. Koraklija and I. Mijatovic. "Treatment of beverage production wastewater by membrane bioreactor". Desalination 246, pp. 285-293. 2009.

[4] J. Boguniewicz-Zablocka, A. Capodaglio and D. Vogel. "Analysis of wastewater treatment efficiency in a soft drinks industry". E3S Web Conferences, p. 19. DOI: 10.1051/ e3sconf/20171902014, 2017.

[5] M.A. Abdel-Fatah, H.O. Sherif and S.I. Hawash. "Design parameters of waste effluent treatment unit from beverages production".
Ain Shams Engineering Journal, pp. 305-310. 2017.

[6] B.L. Brazil. "Performance and operation of a rotating biological contactor in a tilapia recirculating aquaculture system". Aquacultural engineering. Vol. 34, pp. 261274. 2006.

[7] V. Droppelmann, R. López y M. Wilkes. "Evaluación de una Planta de Tratamiento de Aguas Servidas en Base a Biodiscos". XXVII Congresso Interamericano de Engenharia Sanitária e Ambiental. Associação Brasileira de Engenharia Sanitária e Ambiental ABES. Brasil. 2001.

[8] P. Torres-Lozada, N. Vásquez-Sarria, A. Pérez-Vidal, C.A. Madera- Parra y J.A. Rodríguez-Victori. "Alternativas de Tratamiento Biológico Aerobio Para el Agua Residual Domestica del Municipio de Cali, Colombia". Afinidad LXVIII, 555, pp. 381388. 2011.

[9] C.A. Arenas y M.A. Jaramillo. "Planteamiento y evaluación de un tratamiento biológico para agua residual proveniente de proceso de minería aurífera", pp. 125. 2015.

[10] A. Jacomo, J. López y P. Ures. "Plantas de Tratamiento de efluentes líquidos de la industria textil", pp. 17. 2015.

[11] IDEAM. "Sistemas de gestión de calidad". 10 Septiembre 2007. Available: http://www. ideam.gov.co/documents/14691/38158/ Toma_Muestras_AguasResiduales.pdf/ f5baddf0-7d86-4598-bebd-0e123479d428

[12] S. Cortez, P. Teixeira, R. Oliveira and M. Mota, "Rotating biological contactors: a review on main factors affecting performance". Rev. Environ. Sci. Biotechnol. Vol. 7, pp. 155-172. 2008.

[13] A.D. Andreadakis. "Design of multi-stage Rotating Biological Contactors". J. Envir. Eng. Div. ASCE, 113(EE1), pp. 199-205. 1987.

[14] R.L. Antoine. "Design Criteria for application of the Rotating Biological Contactor to domestic and industrial wastewater treatment". Proc. Int. Env. Colloquium, Liege, Belgium. 1978.

[15] WSP. "Biofiltro: Una opción sostenible para el tratamiento de aguas residuales en pequeñas localidades". Banco Mundial. 2006.

[16] D. Peña y P. Cisterna. "Determinación de la relación $\mathrm{DQO} / \mathrm{DBO}_{5}$ en aguas residuales 
de comunas con población menor a 25.000 habitantes en la VIII región". Universidad Técnica Federico Santa María. Valparaíso, Chile.

[17] Metcalf and Eddy. "Wastewater engineering, Treatment and reuse". McGraw-Hill, New York. 2003.

[18] G. Moeller y A. Tomasini. "Microbiología de Lodos Activados". Instituto de Hidrología, Metereología y Estudios Ambientales IDEAM. Bogotá, Colombia. 2004.

[19] M. Von Sperling. "Activated sludge and anaerobic biofilm reactors wastewater treatment". IWA Publishing. 2007.

[20] A. Tawflik, H. Temmink, G. Zeeman and B. Klapwijk. "Sewage treatment in a Rotating
Biological Contactor (RBC) System”. Water, Air and Soil Pollution, pp. 275-289. 2006.

[21] M. Martin Cereceda, S. Serrano and A. Guinea. "Biofilm Communities and operational monitoring of a Rotating Biological Contactor System". Water, Air and Soil Pollution, pp. 193-206. 2001.

[22] S.V. Pylink and I.G. Dueck. "Startup simulation for a Rotating Biological Contactor". Theoretical Foundations of Chemical Engineering, pp. 72-79. 2012.

[23] M.Y. Chen and M.J. Syu. "Film analysis of activated sludge microbial discs by the Taguchi method and grey relational analysis". Bioprocess Biosyst Eng, pp. 83-92. 2003. 